\title{
A SCHUR-COHN THEOREM FOR MATRIX POLYNOMIALS
}

\author{
by HARRY DYM and NICHOLAS YOUNG
}

(Received 14th July 1988)

\begin{abstract}
Let $N(\lambda)$ be a square matrix polynomial, and suppose det $N$ is a polynomial of degree $d$. Subject to a certain non-singularity condition we construct a $d$ by $d$ Hermitian matrix whose signature determines the numbers of zeros of $N$ inside and outside the unit circle. The result generalises a well known theorem of Schur and Cohn for scalar polynomials. The Hermitian "test matrix" is obtained as the inverse of the Gram matrix of a natural basis in a certain Krein space of rational vector functions associated with $N$. More complete results in a somewhat different formulation have been obtained by Lerer and Tismenetsky by other methods.
\end{abstract}

1980 Mathematics subject classification (1985 Revision): 30C15, 46D05, 93D20.

\section{Introduction}

A celebrated paper of $I$. Schur [11] contains, among much else, a criterion for a polynomial $p(\lambda)$ to have all its zeros in the open unit disc $\mathbb{D}$ : one forms a certain Hermitian matrix $H$ from the coefficients of $p$, and the assertion is that the zeros of $p$ lie in $\mathbb{D}$ if and only if $H$ is positive definite. The theorem was generalized by A. Cohn [4]: if the zeros of $p$ are all non-conjugate with respect to the unit circle $\mathbb{T}$ then the numbers of zeros inside and outside the unit circle are equal to the numbers of positive and negative eigenvalues of $H$ respectively. The corresponding problem for polynomial matrices holds considerable interest, both for its own sake and for application to multivariable systems theory: the stability of a linear system with many inputs and outputs depends on the zeros of an associated matrix polynomial lying in $\mathbb{D}$. Now the zeros of a square matrix polynomial $N(\lambda)$ (also known as the eigenvalues of $N$ ) are defined to be the zeros of the scalar polynomial det $N(\lambda)$, and so one way of testing $N$ is to apply Schur's criterion to det $N$. However, since the calculation of the determinant of a matrix polynomial is lengthy and numerically somewhat ill behaved, it is natural to look for a more direct way of associating with $N(\lambda)$ a Hermitian matrix whose signature indicates the location of the zeros of $N$ with respect to $\pi$.

One way to approach this question is through Bezoutian matrices. In the scalar case the Schur matrix can be regarded as the Bezoutian of two polynomials, and there are several extensions of the notion of Bezoutian to matrix polynomials. The most successful one appears to be that due to Anderson and Jury [2], who asked whether their form of Bezoutian could be used to prove an analogue of the Schur-Cohn 
theorem. Lerer and Tismenetsky in their very interesting paper [9] proved that the answer was yes. However, their matrix polynomial version of the Schur-Cohn test differs from the original in two significant respects. The first is that, whereas the Schur matrix can be written down directly in terms of the coefficients of the polynomial, in the matrix case one must first construct a certain derived matrix polynomial which is itself difficult to carry out. The second point of difference relates to the size of the "test matrices". The Schur matrix corresponding to a scalar polynomial of degree $n$ is $n \times n$, so that it has the same number of eigenvalues as the polynomial has zeros. In the generalization in [9] the Schur-type matrix corresponding to an $m \times m$ matrix polynomial $N(\lambda)$ is an $m k \times m k$ Hermitian matrix for some integer $k$, whereas the degree of det $N(\lambda)$ may be smaller than $m k$.

In this paper we prove a different generalization of the Schur-Cohn theorem. Although our method is different, we arrive at a result which is similar to that of [9], and even coincident with it in the case of monic matrix polynomials whose zeros are non-conjugate with respect to the unit circle. In the non-monic case, if $N(\lambda)$ is column reduced and det $N(\lambda)$ has degree $d$, then the test matrix we derive is $d \times d$, so that equality between the numbers of zeros of $N$ and eigenvalues of the test matrix is preserved. The price is that $N$ must be brought to column reduced form. This can involve a non-trivial amount of effort (although the property of being column reduced is generic). We obtain our result through a geometric interpretation of the test matrix: it is the inverse of the Gram matrix of a natural basis in a certain indefinite inner product space of rational vector functions associated with $N$. This interpretation provides extra information even in the scalar case. Both approaches have their merits: the treatment in [9] is based on the spectral theory of matrix polynomials, as presented in [7], and is strictly finite-dimensional throughout. Ours brings in the infinite-dimensional spaces $L^{2}$ and $H^{2}$ of functions on the unit circle, and may be more accessible to those who are familiar with analysis in these spaces. We also use some elements of the theory of indefinite inner product spaces with reproducing kernel.

We recapitulate some standard terminology from [8]. Consider an $m \times m$ matrix polynomial $N(\lambda) . N$ is said to be nonsingular if its determinant is not identically zero. The degree of the $j$ th column of $N$ is defined to be the exponent of the highest power of $\lambda$ occurring in that column with nonzero coefficient. Let the $j$ th column of $N$ have degree $d_{j}$ : then the leading column coefficient matrix $N_{0}$ of $N$ is the constant matrix whose $j$ th column is the coefficient of $\lambda^{d_{j}}$ in the $j$ th column of $N(\lambda) . N$ is said to be column reduced if $N_{0}$ is nonsingular. It is easily seen that an equivalent condition is that $\operatorname{det} N(\lambda)$ is a polynomial of degree $\sum_{j} d_{j}$. Any nonsingular $N(\lambda)$ can be reduced by elementary column operations to a column reduced polynomial, without changing its zeros. If $N$ is column reduced, then $\operatorname{det} N(\lambda)$ has degree

$$
d=\sum_{j=1}^{m} d_{j}
$$

and so $N(\lambda)$ has $d$ zeros (we always count the zeros of $N$ according to their multiplicity as zeros of $\operatorname{det} N$ ). 
To state the main theorem we need to describe some matrix polynomials associated with $N$. For any nonsingular $m \times m$ matrix polynomial $N$ with column degrees $d_{1}, \ldots, d_{m}$ we can write

$$
N(\lambda)=\left(N_{0}+N_{1} \lambda^{-1}+\cdots+N_{k} \lambda^{-k}\right) \Delta(\lambda)
$$

where $k=\max _{j} d_{j}$ and

$$
\Delta(\lambda)=\operatorname{diag}\left\{\lambda^{d_{1}}, \ldots, \lambda^{d_{m}}\right\}
$$

Let

$$
\hat{N}(\lambda)=N_{0}^{*}+N_{1}^{*} \lambda+\cdots+N_{k}^{*} \lambda^{k}
$$

An alternative description is

$$
\hat{N}(\lambda)=\Delta(\lambda) N^{\#}(\lambda)
$$

where, for any matrix function $A(\lambda)$,

$$
A^{\#}(\lambda)=A\left(1 / \lambda^{*}\right)^{*}
$$

Superscript star denotes conjugate transpose for matrices and complex conjugation for scalars. Following engineering custom, we shall say that a rational $m \times m$ matrix valued function $F$ is an allpass function if $F$ is both analytic and unitary on $\pi$. Let $N$ be a nonsingular $m \times m$ matrix polynomial. We shall say that an $m \times m$ matrix polynomial $D$ is a reflection of $N$ if (1) $D(0)$ is invertible, (2) $N D^{-1}$ is an allpass function, and (3) $N$ and $D$ are right coprime.

We shall see below that a necessary condition (Theorem 4.1) for $N$ to admit a reflection is that its zeros are off $\pi$ whereas a sufficient condition (Theorem 2.1) is that

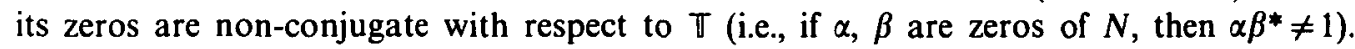
The main conclusion of this paper can be summarized as follows:

Theorem 1.1 Let $N$ be a nonsingular column reduced $m \times m$ matrix polynomial with column degrees $d_{1}, \ldots d_{m}$ which admits a reflection $D$ and let $\hat{N}=\Delta N^{\#}, \hat{D}=\Delta D^{\#}$, where

$$
\Delta(\lambda)=\operatorname{diag}\left\{\lambda^{d_{1}}, \ldots, \lambda^{d_{m}}\right\}
$$

Then $\hat{N}, \hat{D}$ are matrix polynomials which can be written

$$
\begin{aligned}
& \hat{N}(\lambda)=N_{0}^{*}+N_{1}^{*} \lambda+\cdots+N_{k}^{*} \lambda^{k}, \\
& \hat{D}(\lambda)=D_{0}^{*}+D_{1}^{*} \lambda+\cdots+D_{k}^{*} \lambda^{k},
\end{aligned}
$$


where $k=\max _{j} d_{j}$. Let $d=\sum_{j=1}^{m} d_{j}$ and let $X, Y$ be the $d \times m k$ matrices which are obtained from the block Toeplitz matrices

$$
\left[\begin{array}{cccc}
N_{0}^{*} & 0 & \ldots & 0 \\
N_{1}^{*} & N_{0}^{*} & \ldots & 0 \\
\vdots & \vdots & \vdots & \vdots \\
N_{k-1}^{*} & N_{k-2}^{*} & \ldots & N_{0}^{*}
\end{array}\right],\left[\begin{array}{cccc}
D_{0}^{*} & 0 & \ldots & 0 \\
D_{1}^{*} & D_{0}^{*} & \ldots & 0 \\
\vdots & \vdots & \vdots & \vdots \\
D_{k-1}^{*} & D_{k-2}^{*} & \ldots & D_{0}^{*}
\end{array}\right],
$$

respectively, by the deletion of the $c$-th row in block row $p(0 \leqq p \leqq k-1,1 \leqq c \leqq m)$ whenever $p \geqq d_{c}$. Then the number of zeros of $N$ inside and outside $\mathbb{T}$ are respectively equal to the number of positive and negative eigenvalues of the invertible Hermitian matrix $X X^{*}-Y Y^{*}$.

The reader may check that if $N$ is a scalar polynomial, say

$$
N(\lambda)=a_{0}+a_{1} \lambda+\cdots+a_{k} \lambda^{k}, \quad a_{k} \neq 0
$$

then

$$
\widehat{N}(\lambda)=a_{k}^{*}+a_{k-1}^{*} \lambda+\cdots+a_{0}^{*} \lambda^{k}
$$

and $\hat{D}=N$, so that in this case

$$
X=\left[\begin{array}{cccc}
a_{k}^{*} & 0 & \ldots & 0 \\
a_{k-1}^{*} & a_{k}^{*} & \ldots & 0 \\
\vdots & \vdots & \vdots & \vdots \\
a_{1}^{*} & a_{2}^{*} & \ldots & a_{k}^{*}
\end{array}\right], \quad Y=\left[\begin{array}{cccc}
a_{0} & 0 & \ldots & 0 \\
a_{1} & a_{0} & \ldots & 0 \\
\vdots & \vdots & \vdots & \vdots \\
a_{k-1} & a_{k-2} & \ldots & a_{0}
\end{array}\right]
$$

and we recover the Schur-Cohn theorem. Unfortunately, if $N$ is a matrix polynomial there is no such simple recipe for the entries of $Y$ in terms of the coefficients of $N$. The rational interpolation theory of [3] gives a way of constructing $D$ (using state space methods) in terms of the solution of a linear matrix equation of the Stein type. However, if one has to solve such equations in order to invoke the theory developed here, then the total effort becomes impractical and presumably one could do as well by resorting to an appropriate inertia theorem.

We prove Theorem 1.1 in Section 4 below. The proof uses some elements of the theory of indefinite inner product spaces of functions with a reproducing kernel: these are described in Section 3. The functions in question belong to $L_{m}^{2}$, the space of square integrable $\mathbb{C}^{m}$-valued functions on $\mathbb{T}$, with inner product

$$
<f, g\rangle=\frac{1}{2 \pi} \int_{0}^{2 \pi} g\left(e^{i \theta}\right)^{*} f\left(e^{i \theta}\right) d \theta .
$$


The basic idea is to introduce a subspace $\mathscr{K}(\Theta)$ of $L_{m}^{2}$ based on the allpass function $\Theta=N D^{-1}$, where $D$ is a reflection of $N . \mathscr{K}(\Theta)$, endowed with an appropriately defined indefinite inner product $[,]_{\mathscr{X}}$, is then a finite dimensional reproducing kernel Krein space which splits into the direct sum of two subspaces, one positive and one negative with respect to $[,]_{\mathscr{r}}$. The dimension of the positive space is equal to the number of zeros of $N$ inside $T$ whereas the dimension of the negative space is equal to the number of zeros of $N$ outside $T$. The final recipe is obtained by identifying the Gram matrix of a natural basis of $\mathscr{K}(\Theta)$ with respect to $[,]_{\mathscr{X}}$ as $\left(X X^{*}-Y Y^{*}\right)^{-1}$.

Since the detailed analysis for matrix polynomials is somewhat lengthy, it seems worthwhile to sketch the method on the simple scalar example

$$
N(\lambda)=\left(\lambda-\frac{1}{2}\right)(\lambda+3)(\lambda-4)
$$

For this choice of $N$ it is readily checked that

$$
D(\lambda)=\left(1-\frac{1}{2} \lambda\right)(1+3 \lambda)(1-4 \lambda)
$$

is a reflection and that correspondingly

$$
\Theta(\lambda)=N(\lambda) D(\lambda)^{-1}=\Psi_{1}(\lambda)^{-1} \Psi_{2}(\lambda)
$$

where

$$
\Psi_{1}(\lambda)=\frac{\left(\lambda+\frac{1}{3}\right)\left(\lambda-\frac{1}{4}\right)}{\left(1+\frac{1}{3} \lambda\right)\left(1-\frac{1}{4} \lambda\right)} \quad \text { and } \quad \Psi_{2}(\lambda)=\frac{\lambda-\frac{1}{2}}{1-\frac{1}{2} \lambda}
$$

are Blaschke products which are associated with the zeros of $N$ outside and inside $\mathbb{D}$, respectively. For each such product $\Psi_{j}=N_{j} / D_{j}$ there is an associated backwards shift invariant subspace

$$
\mathscr{H}\left(\Psi_{j}\right)=H^{2} \ominus \Psi_{j} H^{2}=\left\{\frac{p}{D_{j}}: \text { degree } p<\text { degree } D_{j}\right\}
$$

of the Hardy space $H^{2}$.

We now set

$$
\mathscr{K}(\Theta)=\left\{\frac{p}{D}: \text { degree } p<3\right\}
$$

and observe that $\Psi_{1} \mathscr{K}(\Theta)$ is the direct sum of the two-dimensional space 


$$
\Psi_{1} \mathscr{K}_{1}=\Psi_{1}\left\{\frac{p(\lambda)}{(1+3 \lambda)(1-4 \lambda)}: \text { degree } p<2\right\}=\mathscr{H}\left(\Psi_{1}\right)
$$

and the one dimensional space

$$
\Psi_{1} \mathscr{K}_{2}=\left\{\frac{p(\lambda)}{1-\frac{1}{2} \lambda}: \text { degree } p<1\right\}=\mathscr{H}\left(\Psi_{2}\right)
$$

Thus every $f$ [resp. $g]$ in $\mathscr{K}(\Theta)$ admits a unique decomposition

$$
f=f_{1}+f_{2} \quad\left[\text { resp. } g=g_{1}+g_{2}\right]
$$

with $f_{i}$ [resp. $g_{i}$ in $\mathscr{K}_{i}$. Correspondingly we define

$$
[f, g]_{\mathscr{X}}=\left\langle f_{2}, g_{2}\right\rangle-\left\langle f_{1}, g_{1}\right\rangle
$$

Then, for any basis $e_{1}, e_{2}, e_{3}$ of $\mathscr{K}(\Theta)$, the Gram matrix $\left[\left[e_{j}, e_{i}\right]_{\mathscr{X}}\right]$ is a $3 \times 3$ Hermitian matrix with one positive and two negative eigenvalues. Moreover, the space $\mathscr{K}(\Theta)$, with this indefinite inner product is a reproducing kernel Krein space with exactly one reproducing kernel which can be expressed both as

$$
K_{\omega}(\lambda)=\frac{1-\Theta(\lambda) \Theta(\omega)^{*}}{1-\omega^{*} \lambda}
$$

and as

$$
K_{\omega}(\lambda)=\sum_{i, j=1}^{3} e_{i}(\lambda)\left(G^{-1}\right)_{i j} e_{j}(\omega)^{*}
$$

for any basis $e_{1}, e_{2}, e_{3}$ of $\mathscr{K}(\Theta)$. The Gram matrix corresponding to the particular basis

$$
e_{j}(\lambda)=\lambda^{j-1} / D(\lambda), \quad j=1,2,3
$$

is the inverse of the Schur matrix $X X^{*}-Y Y^{*}$ with $X$ and $Y$ specified in terms of the coefficients $a_{0}, \ldots, a_{3}$ of $N(\lambda)$ as indicated in the discussion of the scalar polynomial following the statement of Theorem 1.1. This emerges by matching the two stated expressions for the reproducing kernel with the special basis $e_{j}(\lambda)=\lambda^{j-1} / D(\lambda)$ in the latter and leads ultimately to the conclusion that (in this example) the Schur matrix has one positive and two negative eigenvalues.

The argument generalizes to matrix polynomials, but with technical complications. In particular it is no longer so simple to find a reflection $D$ such that $N D^{-1}$ is allpass, nor, 
as we have already remarked, is there a simple recipe for the entries in the block matrix $Y$ in terms of the coefficients of $N$.

We are grateful to I. Gohberg and L. Lerer for useful comments on an earlier version of this paper.

\section{Reflections of matrix polynomials}

In this section we shall present a number of elementary properties of reflections of $N(\lambda)$, when they exist. We shall also establish the existence of reflections of $N(\lambda)$ under the assumption that $N(\lambda)$ has no conjugate roots. This condition is not necessary as the example

$$
\begin{gathered}
N(\lambda)=\operatorname{diag}\left\{\lambda-\frac{1}{2}, \lambda-2\right\} \\
D(\lambda)=\operatorname{diag}\left\{1-\frac{1}{2} \lambda, 1-2 \lambda\right\}
\end{gathered}
$$

clearly indicates. Nevertheless we shall deal first with this case because it is the most transparent.

We shall make use of the elementary $m \times m$ allpass functions

$$
B_{\omega}(\lambda)=\left[\begin{array}{cc}
b_{\omega}(\lambda) & 0 \\
0 & I_{m-1}
\end{array}\right]
$$

wherein

$$
b_{\omega}(\lambda)=\frac{\lambda-\omega}{1-\omega^{*} \lambda}
$$

is the more or less standard Blaschke factor although here we allow $\omega$ to be any point in $\mathbb{C}$ with the understanding that $b_{\omega}(\lambda)=-\omega$ if $|\omega|=1$.

Lemma 2.1. Let $N$ be an $m \times m$ matrix polynomial with

$$
\operatorname{det} N(\lambda)=a\left(\lambda-\alpha_{1}\right) \ldots\left(\lambda-\alpha_{n}\right), \quad n \geqq 0, \quad a \in \mathbb{C} \backslash\{0\} .
$$

Then there exists an $m \times m$ matrix polynomial $D$ such that

$$
\begin{aligned}
\Theta(\lambda) & =N(\lambda) D(\lambda)^{-1} \\
& =V_{0} B_{1}(\lambda) V_{1} B_{2}(\lambda) V_{2} \ldots V_{n-1} B_{n}(\lambda) V_{n},
\end{aligned}
$$

where $V_{0}, \ldots, V_{n}$ are constant $m \times m$ unitary matrices, and $B_{j}=B_{\alpha_{g}}, j=1, \ldots, n$. In particular, the rational matrix function $\Theta(\lambda)$ is an allpass function and 


$$
\operatorname{det} D(\lambda)=b\left(1-\alpha_{1}^{*} \lambda\right) \ldots\left(1-\alpha_{n}^{*} \lambda\right),
$$

where $b$ is a constant with $|b|=|a|$.

Here an empty product (the case $n=0$ ) is to be interpreted as 1 .

Proof. If $n=0$, then $D=N$ does the trick.

Suppose next that $n \geqq 1$. Let $Z$ denote the set of constant $m \times m$ diagonal matrices with a zero in the top left entry. Then, since $\operatorname{det} N\left(\alpha_{1}\right)=0$, there exists a pair of constant $m \times m$ unitary matrices $U_{1}$ and $V_{1}$ such that

$$
U_{1}^{*} N\left(\alpha_{1}\right) V_{1}^{*} \in Z
$$

Thus $N_{1}(\lambda)=U_{1} B_{1}(\lambda)^{-1} U_{1}^{*} N(\lambda)$ is a matrix polynomial with

$$
\operatorname{det} N_{1}(\lambda)=a\left(1-\alpha_{1}^{*} \lambda\right)\left(\lambda-\alpha_{2}\right) \ldots\left(\lambda-\alpha_{n}\right)
$$

Next, since $\operatorname{det} N_{1}\left(\alpha_{2}\right)=0$, there exists a pair of constant $m \times m$ unitary matrices $U_{2}$ and $V_{2}$ such that

$$
U_{2}^{*} N_{1}\left(\alpha_{2}\right) V_{1}^{*} \in Z
$$

and hence

$$
N_{2}(\lambda)=U_{2} B_{2}(\lambda)^{-1} U_{2}^{*} N_{1}(\lambda)
$$

is a matrix polynomial with

$$
\operatorname{det} N_{2}(\lambda)=a\left(1-\alpha_{1}^{*} \lambda\right)\left(1-\alpha_{2}^{*} \lambda\right)\left(\lambda-\alpha_{3}\right) \ldots\left(\lambda-\alpha_{n}\right)
$$

Upon continuing this way for $(n-2)$ more steps it is readily seen that

$$
N_{n}(\lambda)=U_{n} B_{n}(\lambda)^{-1} U_{n}^{*} \ldots U_{1} B_{1}(\lambda)^{-1} U_{1}^{*} N(\lambda)
$$

is a matrix polynomial with

Moreover,

$$
\operatorname{det} N_{n}(\lambda)=a\left(1-\alpha_{1}^{*} \lambda\right) \ldots\left(1-\alpha_{n}^{*} \lambda\right)
$$

$$
N(\lambda)=\Theta(\lambda) N_{n}(\lambda)
$$

where

$$
\Theta(\lambda)=U_{1} B_{1}(\lambda) U_{1}^{*} \ldots U_{n} B_{n}(\lambda) U_{n}^{*},
$$


is clearly an allpass function. The rest is straightforward upon setting

$$
D(\lambda)=N_{n}(\lambda)
$$

Theorem 2.1. Let $N$ be a nonsingular $m \times m$ matrix polynomial whose zeros are nonconjugate with respect to $\mathbb{T}$. Then $N$ admits a reflection $D$.

Proof. By Lemma 2.1 there exists an $m \times m$ matrix polynomial $D$ such that (1) $D(0)$ is invertible and (2) $N D^{-1}$ is an allpass function. The extra assumption that $N$ has no conjugate roots guarantees that $N$ and $D$ are right (and left) coprime.

A point $\alpha \in \mathbb{C}$ is said to be a pole of a rational $m \times m$ matrix valued function $F$ if one of more entries in $F$ has a pole at $\alpha$ (or equivalently if $F$ admits a matrix Laurent expansion $F(\lambda)=\sum_{j=-v}^{\infty} F_{j}(\lambda-\alpha)^{j}$ with matrix coefficients $F_{j}$ where $v \geqq 1$ and $F_{-v}$ is nonzero). A point $\alpha \in \mathbb{C}$ is said to be a zero of a nonsingular rational $m \times m$ matrix valued function $F(\lambda)$ if there exists an $m \times 1$ vector valued polynomial $f$ such that (1) $F f$ is analytic at $\alpha,(2)(F f)(\alpha)=0$ and (3) $f(\alpha) \neq 0$. If $F$ is already analytic at $\alpha$, then it suffices to choose $f=f(\alpha)$ constant. Thus this definition is consistent with the definition introduced earlier for matrix polynomials. Moreover, it is readily checked that a point $\alpha \in \mathbb{C}$ is a zero of a nonsingular rational $m \times m$ matrix function $F$ if and only if it is a pole for $F^{-1}$.

The need for extra care in the definition of a zero of a rational matrix valued function arises because even an allpass function can have both a zero and a pole at the same point $\alpha \in \mathbb{C}$ (neither of which appear in the determinant) as is illustrated by the example

$$
F(\lambda)=\operatorname{diag}\left\{\lambda, \lambda^{-1}\right\}
$$

Lemma 2.2. Let $\Theta$ be a rational $m \times m$ allpass function with constant determinant and suppose that no point $\alpha \in \mathbb{C}$ is both a zero and a pole of $\Theta$. Then $\Theta$ is a constant matrix.

Proof. If $\alpha$ is a zero of $\Theta$, then $\alpha$ is not a pole of $\Theta$ and hence $\Theta$ is analytic at $\alpha$ and $\operatorname{det} \Theta(\alpha)=0$. But this is impossible for an allpass function with constant determinant. Thus $\Theta$ has no zeros in $\mathbb{C}$. The same argument applied to $\Theta^{-1}$ guarantees that $\Theta$ has no poles in $\mathbb{C}$ either, i.e., the entries of $\Theta$ are entire rational functions. Moreover, $\Theta(\lambda)$ is invertible at every point $\lambda \in \mathbb{C}$. Thus the relation

$$
\Theta^{\#}(\lambda)=\Theta(\lambda)^{-1}, \quad \lambda \in \mathbb{T},
$$

which is valid for allpass functions on $\pi$ extends by analytic continuation to all of $\mathbb{C}$. Therefore

$$
\begin{aligned}
\lim _{\lambda \rightarrow \infty} \Theta(\lambda) & =\lim _{\lambda \rightarrow \infty} \Theta^{\#}(\lambda)^{-1} \\
& =\lim _{\lambda \rightarrow \infty}\left\{\Theta\left(1 / \lambda^{*}\right)^{*}\right\}^{-1}
\end{aligned}
$$




$$
=\left\{\Theta(0)^{*}\right\}^{-1}
$$

Hence, the entries in $\Theta$ are bounded entire functions and so, by Liouville's theorem, are all constant.

The example which was presented in the first few lines of this section shows that the condition of nonoverlapping zeros and poles is needed in both the last and the next lemmas.

Lemma 2.3. Let $\Theta$ be a rational $m \times m$ allpass function with

$$
\operatorname{det} \Theta(\lambda)=a b_{\alpha_{1}}(\lambda) \ldots b_{\alpha_{n}}(\lambda)
$$

where $a \in \mathbb{T}, n \geqq 1$ and $\alpha_{i} \alpha_{j}^{*} \neq 1$ for $i, j=1, \ldots, n$ and suppose further that no point $\alpha \in \mathbb{C}$ is both a zero and a pole of $\Theta$. Then the points $\alpha_{1}, \ldots, \alpha_{n}$ are the zeros of $\Theta$ repeated according to multiplicity and there exist constant unitary matrices $V_{0}, \ldots, V_{n}$ such that

$$
\Theta(\lambda)=V_{0} B_{\alpha_{1}}(\lambda) V_{1} B_{\alpha_{2}}(\lambda) \ldots V_{n-1} B_{\alpha_{n}}(\lambda) V_{n}
$$

for every point $\lambda \in \mathbb{C} \backslash\left\{1 / \alpha_{i}^{*}: 1 \leqq i \leqq n\right.$ and $\left.\alpha_{i} \neq 0\right\}$.

Proof. The first step is to check that $\alpha_{1}, \ldots, \alpha_{n}$ are not poles of $\Theta$. Suppose to the contrary that $\alpha_{j}$ is a pole. Then, by assumption, $\alpha_{j}$ is not a zero of $\Theta$, and hence is a zero of $\Theta^{-1}$ and not a pole of $\Theta^{-1}$. But this in turn implies that $\operatorname{det} \Theta^{-1}=\{\operatorname{det} \Theta\}^{-1}$ is analytic and equal to zero at $\alpha_{j}$ which contradicts the presumed form of $\operatorname{det} \Theta$. Thus $\Theta$ is analytic at the points $\alpha_{1}, \ldots, \alpha_{n}$ (some of which may overlap). In fact, the assumption that no point $\alpha \in \mathbb{C}$ is both a zero and a pole of $\Theta$ guarantees that the zeros of $\Theta$ correspond to the zeros of $\operatorname{det} \Theta$ and hence, in the present setting, that the zeros of $\Theta$, repeated according to multiplicity, are precisely the points $\alpha_{1}, \ldots, \alpha_{n}$.

Next, just as in the proof of Lemma 2.1, we can find a unitary matrix $V_{0}$ such that the first row of $V_{0}^{*} \Theta\left(\alpha_{1}\right)$ is equal to zero. Thus the new allpass function

$$
\Theta_{1}(\lambda)=B_{\alpha_{1}}(\lambda)^{-1} V_{0}^{*} \Theta(\lambda)
$$

is analytic at $\alpha_{1}$ with

$$
\operatorname{det} \Theta_{1}(\lambda)=a_{1} b_{\alpha_{2}}(\lambda) \ldots b_{\alpha_{n}}(\lambda), \quad a_{1} \in \mathbb{T}
$$

Continuing this way for $n-1$ more steps we see that

$$
\Theta_{n}(\lambda)=B_{\alpha_{n}}(\lambda)^{-1} V_{n-1}^{*} \ldots B_{\alpha_{1}}(\lambda)^{-1} V_{0}^{*} \Theta(\lambda)
$$

is analytic at $\alpha_{1}, \ldots, \alpha_{n}$ and that its determinant is constant and nonzero. In particular

$$
\operatorname{det} \Theta_{n}\left(\alpha_{j}\right) \neq 0, \quad j=1, \ldots, n,
$$


and hence, since $\Theta_{n}$ is clearly allpass, it further follows by letting $\lambda \rightarrow \alpha_{j}$ in the relation

$$
\Theta_{n}(\lambda) \Theta_{n}^{\#}(\lambda)=I
$$

that $\Theta_{n}$ is analytic with nonzero determinant at every point $1 / \alpha_{j}^{*}, j=1, \ldots, n$, with $\alpha_{j} \neq 0$. It follows readily that $\Theta_{n}$ has no zeros in $\mathbb{C}$ and hence, by Lemma 2.2 ,

$$
\Theta_{n}(\lambda)=V_{n}
$$

a constant unitary matrix. This is, however, clearly equivalent to the asserted formula.

Let $\delta_{i}(P), i=1, \ldots, m$, denote the $i$ th column degree of an $m \times m$ matrix polynomial $P(\lambda)$, i.e., $\delta_{i}(P)$ is the highest power of $\lambda$ which appears in the $i$ th column of $P$.

Lemma 2.4. Let $N$ and $D$ be $m \times m$ matrix polynomials such that $N=\Theta D$, where $\Theta$ is a rational $m \times m$ allpass function which is analytic at zero. Then

$$
\delta_{i}(N) \geqq \delta_{i}(D), \quad 1 \leqq i \leqq m
$$

Proof. Let $e_{i}, i=1, \ldots, m$, denote the $i$ th standard basis vector of $\mathbb{C}^{m}$ and suppose that $\delta_{i}(N)=k$ for some fixed choice of $i$. Then

$$
x=\lim _{\lambda \rightarrow \infty} \frac{N(\lambda)}{\lambda^{k}} e_{i}
$$

is a nonzero vector in $\mathbb{C}^{m}$ and

$$
\begin{aligned}
\lim _{\lambda \rightarrow \infty} \frac{D(\lambda)}{\lambda^{k}} e_{i} & =\lim _{\lambda \rightarrow \infty} \frac{\Theta\left(1 / \lambda^{*}\right)^{*} N(\lambda)}{\lambda^{k}} e_{i} \\
& =\Theta(0)^{*} x .
\end{aligned}
$$

Thus

$$
\delta_{i}(D)=k=\delta_{i}(N) \quad \text { if } \quad \Theta(0)^{*} x \neq 0
$$

whereas

$$
\delta_{i}(D)<k=\delta_{i}(N) \quad \text { if } \quad \Theta(0)^{*} x=0
$$


Lemma 2.5. If $N$ is a nonsingular $m \times m$ matrix polynomial with reflection $D$ and if $\Theta=N D^{-1}$, then

(1) $\hat{N}=\Delta N^{\#}$ and $\hat{D}=\Delta D^{\#}$ are both matrix polynomials, and

(2) $\hat{N}^{-1} \hat{D}=\Theta=N D^{-1}$.

If also $N$ is column reduced, then

(3) $\hat{N}$ and $\hat{D}$ are left coprime.

Proof. It is readily checked that $\hat{N}$ is a matrix polynomial. Since $\delta_{i}(D) \leqq \delta_{i}(N)$, for $i=1, \ldots, m$, by Lemma 2.4 , the same holds true for $\hat{D}$.

Next, since $\Theta=N D^{-1}$ is allpass,

$$
\begin{aligned}
\Theta(\lambda) & =\left\{\Theta^{\#}(\lambda)\right\}^{-1}=\left\{N^{\#}(\lambda)\right\}^{-1} D^{\#}(\lambda) \\
& =\left\{\Delta(\lambda) N^{\#}(\lambda)\right\}^{-1} \Delta(\lambda) D^{\#}(\lambda) \\
& =\hat{N}(\lambda)^{-1} \hat{D}(\lambda)
\end{aligned}
$$

This proves (2).

Finally, if $\hat{N}$ and $\hat{D}$ are not left coprime, then there exists a point $\alpha \in \mathbb{C}$ and a nonzero vector $x \in \mathbb{C}^{m}$ such that

$$
x^{*} \hat{N}(\alpha)=0 \quad \text { and } \quad x^{*} \hat{D}(\alpha)=0
$$

The point $\alpha \neq 0$ because $\hat{N}(0)$ is invertible when $N$ is column reduced. Thus

$$
\left\{x^{*} \hat{N}(\alpha)\right\}^{*}=N\left(1 / \alpha^{*}\right) \Delta(\alpha)^{*} x=0
$$

and

$$
\left\{x^{*} \hat{D}(\alpha)\right\}^{*}=D\left(1 / \alpha^{*}\right) \Delta(\alpha)^{*} x=0,
$$

which contradicts the assumption that $N$ and $D$ are right coprime since

$$
\Delta(\alpha)^{*} x \neq 0
$$

for $\alpha \neq 0$.

Lemma 2.6. Let $N$ be a nonsingular $m \times m$ matrix polynomial whose zeros are nonconjugate with respect to $T$ and let $\Theta=N D^{-1}$, where $D$ is a reflection of $N$. Suppose further that 


$$
\operatorname{det} N(\lambda)=a\left(\lambda-\alpha_{1}\right) \ldots\left(\lambda-\alpha_{n}\right), \quad a \in \mathbb{C} \backslash\{0\}
$$

Then the following hold:

(1) $\Theta$ is analytic at every point $\lambda \in \mathbb{C}$ such that $1-\alpha_{i}^{*} \lambda \neq 0$ for $i=1, \ldots, n$ and hence in particular at the points $\alpha_{1}, \ldots, \alpha_{n}$;

(2) the polynomials $\operatorname{det} N(\lambda)$ and $\operatorname{det} D(\lambda)$ have no common zeros;

$$
\operatorname{det} D(\lambda)=b\left(1-\alpha_{1}^{*} \lambda\right) \ldots\left(1-\alpha_{n}^{*} \lambda\right)
$$

and

$$
\operatorname{det} \Theta(\lambda)=c \prod_{j=1}^{n} \frac{\lambda-\alpha_{j}}{1-\alpha_{j}^{*} \lambda}
$$

for some choice of $b \in \mathbb{C} \backslash\{0\}$ and $c \in \mathbb{T}$, respectively;

(4) no point $\alpha \in \mathbb{C}$ is both a zero and a pole of $\Theta$;

(5) the polynomials $\operatorname{det} \hat{N}(\lambda)$ and $\operatorname{det} \hat{D}(\lambda)$ have no common zeros.

Proof. We may suppose without loss of generality that $N$ is column reduced. Then, by Lemma 2.5 ,

$$
\hat{N}=\Delta N^{\#} \text { and } \hat{D}=\Delta D^{\#}
$$

are matrix polynomials and

$$
\Theta=\hat{N}^{-1} \hat{D}
$$

Thus $\Theta$ is analytic except possibly at the zeros of $\hat{N}$. But, since $N$ is column reduced,

$$
n=d=\sum_{i=1}^{m} d_{i}
$$

and thus it is readily checked that

$$
\operatorname{det} \hat{N}(\lambda)=a^{*} \prod_{j=1}^{n}\left(1-\lambda \alpha_{j}^{*}\right)
$$

Therefore, by the non-conjugacy assumption,

$$
\operatorname{det} \hat{N}\left(\alpha_{i}\right) \neq 0, \quad i=1, \ldots, n,
$$

which serves to establish (1). 
Next, since $N$ and $D$ are right coprime, there exist a pair of $m \times m$ polynomial matrices $A$ and $B$ such that

$$
A(\lambda) N(\lambda)+B(\lambda) D(\lambda)=I
$$

for every point $\lambda \in \mathbb{C}$ (see e.g., [10]). But this in turn implies that

$$
\{A(\lambda) \Theta(\lambda)+B(\lambda)\} D(\lambda)=I
$$

at every point $\lambda \in \mathbb{C}$ in the domain of analyticity of $\Theta$ and hence in particular at the points $\alpha_{1}, \ldots, \alpha_{n}$, by (1). Therefore $D\left(\alpha_{i}\right)$ is invertible. Thus the zeros of $\operatorname{det} D(\lambda)$ do not overlap with the zeros of det $N(\lambda)$, as asserted in (2).

Assertion (3) is immediate if $D$ is a constant matrix. Otherwise, since $D(0)$ is non-singular,

$$
\operatorname{det} D(\lambda)=b\left(1-\beta_{1}^{*} \lambda\right) \ldots\left(1-\beta_{k}^{*} \lambda\right)
$$

for some choice of $b$ and $\beta_{1}, \ldots, \beta_{k}$ in $\mathbb{C} \backslash\{0\}$. Now, as $N D^{-1}$ is allpass, it is readily seen that

$$
1=\frac{|a|^{2}\left(\lambda-\alpha_{1}\right) \ldots\left(\lambda-\alpha_{n}\right)\left(1-\alpha_{1}^{*} \lambda\right) \ldots\left(1-\alpha_{n}^{*} \lambda\right)}{|b|^{2}\left(1-\beta_{1}^{*} \lambda\right) \ldots\left(1-\beta_{k}^{*} \lambda\right)\left(\lambda-\beta_{1}\right) \ldots\left(\lambda-\beta_{k}\right) \lambda^{n-k}}
$$

and hence that

$$
\begin{aligned}
n-k & =\text { the number of terms } \alpha_{j}, j=1, \ldots, n,: \alpha_{j}=0 \\
& \geqq 0
\end{aligned}
$$

and therefore, in view of (2), that

$$
\left(\lambda-\alpha_{1}\right) \ldots\left(\lambda-\alpha_{n}\right)=d\left(\lambda-\beta_{1}\right) \ldots\left(\lambda-\beta_{k}\right) \lambda^{n-k}
$$

for some choice of $d \in \mathbb{C} \backslash\{0\}$. But this in turn implies that

$$
\left(1-\beta_{1}^{*} \lambda\right) \ldots\left(1-\beta_{k}^{*} \lambda\right)=\left(1-\alpha_{1}^{*} \lambda\right) \ldots\left(1-\alpha_{n}^{*} \lambda\right),
$$

from which (3) is now self evident for nonconstant $D$ also.

The formulas

clearly imply that

$$
\Theta=N D^{-1} \quad \text { and } \quad \Theta^{-1}=D N^{-1}
$$

$\{$ the poles of $\Theta\} \subset\{$ the zeros of $D\} \subset\left\{1 / \alpha_{j}^{*}: j=1, \ldots, n\right.$ and $\left.\alpha_{j} \neq 0\right\}$, 
whereas

$$
\left\{\text { the poles of } \Theta^{-1}\right\} \subset\{\text { the zeros of } N\} \subset\left\{\alpha_{1}, \ldots, \alpha_{n}\right\} \text {. }
$$

Therefore, since the two indicated point sets are disjoint by assumption, there is no point $\alpha \in \mathbb{C}$ which is simultaneously a pole of both $\Theta$ and $\Theta^{-1}$. But this is equivalent to (4).

Finally (2.3) and the formula

$$
\operatorname{det} \hat{D}(\lambda)=b^{*}\left(\lambda-\alpha_{1}\right) \ldots\left(\lambda-\alpha_{n}\right)
$$

which follows from (2.2) in much the same way, serve to establish (5).

Theorem 2.2. Let $N$ be a nonsingular $m \times m$ matrix polynomial such that

$$
\operatorname{det} N(\lambda)=a\left(\lambda-\alpha_{1}\right) \ldots\left(\lambda-\alpha_{n}\right) \text {, }
$$

where $n \geqq 0$ and $\alpha_{1}, \ldots, \alpha_{n}$ are non-conjugate with respect to $T$ and let $D$ be a reflection of $N$. Then there exist unitary matrices $V_{0}, \ldots, V_{n}$ such that

$$
N(\lambda) D(\lambda)^{-1}=V_{0} B_{\alpha_{1}}(\lambda) V_{1} B_{\alpha_{2}}(\lambda) V_{2} \ldots B_{\alpha_{n}}(\lambda) V_{n}
$$

for all $\lambda \in \mathbb{C} \backslash\left\{1 / \alpha_{i}^{*}: 1 \leqq i \leqq n, \alpha_{i} \neq 0\right\}$.

Proof. Let $\Theta=N D^{-1}$. By Lemma 2.6 no point of $\mathbb{C}$ is both a zero and a pole of $\Theta$, and

$$
\operatorname{det} \Theta(\lambda)=c \prod_{i=1}^{n} \frac{\lambda-\alpha_{i}}{1-\alpha_{i}^{*} \lambda}
$$

(the product is defined to be 1 if $n=0$ ). Hence Lemma 2.3 applies to give the stated conclusion.

\section{The reproducing kernel Krein space $\mathscr{K}(\Theta)$}

In the preceding section we associated a rational $m \times m$ matrix function $\Theta(\lambda)$ which is both analytic and unitary on $\mathbb{T}$ with every suitably restricted matrix polynomial $N(\lambda)$. Now, for each such $\Theta$ we introduce a finite dimensional Krein space $\mathscr{K}(\Theta)$ whose geometry is linked to the locations of the eigenvalues of $N(\lambda)$. The elements of $\mathscr{K}(\Theta)$ are $m \times 1$ rational vector valued functions which are analytic in $\Omega$, the domain of analyticity of $\Theta$ in $\mathbb{D}$. Moreover, $\mathscr{K}(\Theta)$ is a reproducing kernel space: that is to say, there exists an $m \times m$ matrix valued function $K_{\omega}(\lambda)$ defined on $\Omega \times \Omega$ such that, for every choice of $\omega \in \Omega$, $v \in \mathbb{C}^{m}$ and $f \in \mathscr{K}(\Theta)$

(1) $K_{\omega} v \in \mathscr{K}(\Theta)$ (as a function of $\lambda$ ) and 
(2) $\left[f, K_{\omega} v\right]_{\mathscr{X}}=v^{*} f(\omega)$,

wherein $[,]_{\mathscr{X}}$ denotes the indefinite inner product for $\mathscr{K}$. The fact that the reproducing kernel for such a space is unique and can be written in a number of different ways (which must therefore match) will be used to advantage in the sequel. The main result of this section: Theorem 3.1, is adapted from the more general Theorem 6.6 of [1]. For the convenience of the reader we shall present an independent proof. We shall also formulate the theorem itself directly in terms of $\Theta$ itself and not in terms of its factorization

$$
\Theta=\Psi_{1}^{-1} \Psi_{2}
$$

as the "ratio" of a pair of left inner coprime finite Blaschke-Potapov products, $\Psi_{1}$ and $\Psi_{2}$.

The notation

$$
\rho_{\omega}(\lambda)=1-\lambda \omega^{*}
$$

will prove useful.

Theorem 3.1. Let $\Theta$ be a rational $m \times m$ allpass function and let $\Omega$ denote its domain of analyticity in $\mathbb{D}$. Then:

(1) The space

$$
\mathscr{K}(\Theta)=\left(H_{m}^{2}+\Theta H_{m}^{2}\right) \Theta\left(H_{m}^{2} \cap \Theta H_{m}^{2}\right)
$$

is a finite dimensional subspace of $L_{m}^{2}(\mathbb{T})$ consisting of $m \times 1$ vector valued rational functions which are analytic in $\Omega$.

(2) The intersection $\mathscr{K}_{1} \cap \mathscr{K}_{2}$ of the subspaces

$$
\mathscr{K}_{1}=\left(H_{m}^{2}+\Theta H_{m}^{2}\right) \Theta H_{m}^{2}
$$

and

$$
\mathscr{K}_{2}=\left(H_{m}^{2}+\Theta H_{m}^{2}\right) \Theta \Theta H_{m}^{2}
$$

is equal to zero.

(3) The space $\mathscr{K}=\mathscr{K}(\Theta)$ is the direct sum of $\mathscr{K}_{1}$ and $\mathscr{K}_{2}$ :

$$
\mathscr{K}=\mathscr{K}_{1}+\mathscr{K}_{2}
$$

(4) The space $\mathscr{K}=\mathscr{K}(\Theta)$ endowed with the indefinite inner product

$$
[f, g]_{x}=\left\langle f_{2}, g_{2}\right\rangle-\left\langle f_{1}, g_{1}\right\rangle
$$


where the $f_{i}$ and $g_{i}$ are the components of $f$ and $g$ respectively relative to the direct sum decomposition of (3), is a finite dimensional reproducing kernel Krein space with reproducing kernel

$$
K_{\omega}(\lambda)=\frac{I_{m}-\Theta(\lambda) \Theta(\omega)^{*}}{\rho_{\omega}(\lambda)} .
$$

(5) The MacMillan degree of $\Theta$ is equal to the dimension of $\mathscr{K}(\Theta)$.

It is convenient to first establish three preliminary lemmas. The notation

$$
\mathscr{H}(\Psi)=H_{m}^{2} \ominus \Psi H_{m}^{2},
$$

for $m \times m$ matrix inner functions $\Psi$, will be useful in the sequel.

Lemma 3.1. Let $\Psi$ be an $m \times m$ matrix valued Blaschke-Potapov product of length $n$ : that is,

$$
\Psi(\lambda)=V_{0} B_{1}(\lambda) V_{1} B_{2}(\lambda) V_{2} \ldots B_{n}(\lambda) V_{n}
$$

where each $V_{j}$ is a constant $m \times m$ unitary matrix and $B_{j}=\operatorname{diag}\left\{b_{\alpha_{j}}, 1, \ldots, 1\right\}$ with $\left|\alpha_{j}\right|<1$. Then $\mathscr{H}(\Psi)$ is a space of dimension $n$ consisting of rational vector functions.

Proof. Let $\Psi_{j}=V_{j-1} B_{j}$. Then it is readily checked that $\mathscr{H}\left(\Psi_{j}\right)$ is a reproducing kernel Hilbert space wth respect to the standard inner product with kernel

$$
\begin{aligned}
\frac{I_{m}-\Psi_{j}(\lambda) \Psi_{j}(\omega)^{*}}{\rho_{\omega}(\lambda)} & =V_{j-1} \frac{\left\{I_{m}-B_{j}(\lambda) B_{j}(\omega)^{*}\right\}}{\rho_{\omega}(\lambda)} V_{j-1}^{*} \\
& =\frac{V_{j-1} C_{j} V_{j-1}^{*}}{\rho_{\alpha j}(\lambda)}
\end{aligned}
$$

where $C_{j}$ is a constant $m \times m$ matrix of rank 1 . Thus $\mathscr{H}\left(\Psi_{j}\right)$ is a one-dimensional space. The final conclusion drops out from the decomposition

$$
\begin{aligned}
\mathscr{H}(\Psi) & =\mathscr{H}\left(\Psi_{1} \Psi_{2} \ldots \Psi_{n}\right) \\
& =\mathscr{H}\left(\Psi_{1}\right) \oplus \Psi_{1} \mathscr{H}\left(\Psi_{2}\right) \oplus \cdots \oplus \Psi_{1} \ldots \Psi_{n-1} \mathscr{H}\left(\Psi_{n}\right)
\end{aligned}
$$

Lemma 3.2. If $\Theta=\Psi_{1}^{-1} \Psi_{2}$, where $\Psi_{1}$ and $\Psi_{2}$ are finite Blaschke-Potapov products which are both analytic in $\overline{\mathbb{D}}$ and left inner coprime, then the following holds true:

(1) $\mathscr{H}\left(\Psi_{1}\right)+\mathscr{H}\left(\Psi_{2}\right)=H_{m}^{2} \Theta\left(\Psi_{1} H_{m}^{2} \cap \Psi_{2} H_{m}^{2}\right)$

(2) $\Psi_{1}^{-1}\left\{\mathscr{H}\left(\Psi_{1}\right)+\mathscr{H}\left(\Psi_{2}\right)\right\}=\Psi_{1}^{-1} H_{m}^{2} \Theta\left(H_{m}^{2} \cap \Theta H_{m}^{2}\right)$ 
(3) $\Psi_{1}^{-1} H_{m}^{2}=H_{m}^{2}+\Theta H_{m}^{2}$

(4) $H_{m}^{2}+\Theta H_{m}^{2}$ is a closed subspace of $L_{m}^{2}$

(5) $\Psi_{1}^{-1} \mathscr{H}\left(\Psi_{1}\right)=\left(H_{m}^{2}+\Theta H_{m}^{2}\right) \Theta H_{m}^{2}=\mathscr{K}_{1}$

(6) $\Psi_{1}^{-1} \mathscr{H}\left(\Psi_{2}\right)=\left(H_{m}^{2}+\Theta H_{m}^{2}\right) \ominus \Theta H_{m}^{2}=\mathscr{K}_{2}$

(7) $\mathscr{H}\left(\Psi_{1}\right) \cap \mathscr{H}\left(\Psi_{2}\right)=0$.

Proof. (1) is an easy consequence of the general fact that if $\mathscr{M}_{1}$ and $\mathscr{M}_{2}$ are subspaces (closed or not) of a Hilbert space $\mathscr{H}$, then

$$
\left(\mathscr{M}_{1}+\mathscr{M}_{2}\right)^{\perp}=\mathscr{M}_{1}^{\perp} \cap \mathscr{M}_{2}^{\perp}
$$

with $\mathscr{M}_{i}=\mathscr{H}\left(\Psi_{i}\right), i=1,2$. In this instance

$$
\left\{\left(\mathscr{M}_{1}+\mathscr{M}_{2}\right)^{\perp}\right\}^{\perp}=\overline{\mathscr{M}_{1}+\mathscr{M}_{2}}=\mathscr{M}_{1}+\mathscr{M}_{2}
$$

since both of the indicated spaces are finite dimensional.

(2) is an immediate consequence of (1) and the general fact that, for any choice of subspaces $A \supset B, C$ and $D$ in $L_{m}^{2}$,

$$
\Psi_{1}^{-1}(A \ominus B)=\left(\Psi_{1}^{-1} A\right) \Theta\left(\Psi_{1}^{-1} B\right)
$$

and

$$
\Psi_{1}^{-1}(C \cap D)=\left(\Psi_{1}^{-1} C\right) \cap\left(\Psi_{1}^{-1} D\right)
$$

The first of these two identities exploits the fact that $\Psi_{1}$ is inner, the second does not.

Next, the fact that $\Psi_{1}$ and $\Psi_{2}$ are left inner coprime guarantees the existence of a pair of matrix valued functions $X$ and $Y$ in $H_{m \times m}^{\infty}$ such that

$$
\Psi_{1} X+\Psi_{2} Y=I_{m}
$$

a.e. on $\mathbb{T}$; see e.g. Fuhrmann [6] or Dewilde [5]. (In fact the identity extends to the full disc by either Cauchy's formula or the Poisson formula.) Therefore

$$
\begin{aligned}
\Psi_{1}^{-1} H_{m}^{2} & =\Psi_{1}^{-1}\left(\Psi_{1} X+\Psi_{2} Y\right) H_{m}^{2} \\
& =(X+\Theta Y) H_{m}^{2} \\
& \subset H_{m}^{2}+\Theta H_{m}^{2}
\end{aligned}
$$

This guarantees the validity of (3) since the opposite inclusion is self evident.

(4) is an easy consequence of (3), since $\Psi_{1}$ is inner. 
(5) and (6) are immediate from (3) and (3.4).

Finally, it follows from (3.3) that

$$
\mathscr{H}\left(\Psi_{1}\right) \cap \mathscr{H}\left(\Psi_{2}\right)=\left(\Psi_{1} H_{m}^{2}+\Psi_{2} H_{m}^{2}\right)^{\perp}
$$

However, since $\Psi_{1}$ and $\Psi_{2}$ are left inner coprime, it follows further from (3.6) that

$$
\Psi_{1} H_{m}^{2}+\Psi_{2} H_{m}^{2} \supset \Psi_{1} X H_{m}^{2}+\Psi_{2} Y H_{m}^{2}=H_{m}^{2}
$$

and hence that (7) holds.

We remark that (7) is really a consequence of the more general identity

$$
\overline{\Psi_{3} H_{m}^{2}+\Psi_{4} H_{m}^{2}}=\Psi_{5} H_{m}^{2},
$$

which is valid for any pair of $m \times m$ inner functions $\Psi_{3}$ and $\Psi_{4}$ with greatest common left inner divisor $\Psi_{5}$; see e.g. [5].

Lemma 3.3. If $A, B$ and $C=A+B$ are closed subspaces of a Hilbert space $\mathscr{H}$, then

(1) $C \ominus A \cap B=\overline{C \ominus A+C \ominus B}$

(2) $(C \ominus A) \cap(C \ominus B)=0$.

Proof. Take $\mathscr{H}=C$. Then (1) is immediate from (3.3), while (2) is in any case elementary.

Proof of Theorem 3.1. Let $A=H_{m}^{2}$ and $B=\Theta H_{m}^{2}$. Then $A$ and $B$ are both closed subspaces of $L_{m}^{2}$, as is $C=A+B$, thanks to (4) of Lemma 3.2. Therefore, by Lemma 3.3,

$$
\mathscr{K}=\overline{\mathscr{K}_{1}+\mathscr{K}_{2}} \quad \text { and } \quad \mathscr{K}_{1} \cap \mathscr{K}_{2}=0
$$

But, by Lemma 3.1 and (5) and (6) of Lemma $3.2, \mathscr{K}_{1}$ and $\mathscr{K}_{2}$ are finite dimensional and so (2) and (3) hold. The same identification also serves to establish (1).

Now, to obtain (4), observe first that

$$
K_{\omega}(\lambda) v=\frac{v}{\rho_{\omega}(\lambda)}-\frac{\Theta(\lambda) \Theta(\omega)^{*} v}{\rho_{\omega}(\lambda)}
$$

clearly belongs to $H_{m}^{2}+\Theta H_{m}^{2}$ for every choice of $\omega \in \Omega$ and $v \in \mathbb{C}^{m}$. Moreover, if $g \in H_{m}^{2} \cap \Theta H_{m}^{2}$, then

$$
g=\Theta h
$$

for some $h \in H_{m}^{2}$ and therefore, by Cauchy's formula for $H_{m}^{2}$, 


$$
\begin{aligned}
\left\langle g, K_{\omega} v\right\rangle & =\left\langle g, \frac{v}{\rho_{\omega}}\right\rangle-\left\langle\Theta_{h}, \frac{\Theta \Theta(\omega)^{*} v}{\rho_{\omega}}\right\rangle \\
& =v^{*} g(\omega)-\left\langle h, \frac{\Theta(\omega)^{*} v}{\rho_{\omega}}\right\rangle \\
& =v^{*} g(\omega)-v^{*} \Theta(\omega) h(\omega) \\
& =0 .
\end{aligned}
$$

This proves that $K_{\omega} v \in \mathscr{K}(\Theta)$.

Now, by the factorization (3.1),

$$
K_{\omega}(\lambda)=\Psi_{1}(\lambda)^{-1}\left\{k_{\omega}^{2}(\lambda)-k_{\omega}^{1}(\lambda)\right\} \Psi_{1}(\omega)^{*-1},
$$

where

$$
k_{\omega}^{j}(\lambda)=\frac{I_{m}-\Psi_{j}(\lambda) \Psi_{j}(\omega)^{*}}{\rho_{\omega}(\lambda)}
$$

is the reproducing kernel for $\mathscr{H}\left(\Psi_{j}\right), j=1,2$. Therefore, since

$$
\mathscr{K}_{j}=\Psi_{1}^{-1} \mathscr{H}\left(\Psi_{j}\right), \quad j=1,2,
$$

it follows that

$$
K_{\omega}^{1} v=-\Psi_{1}^{-1} k_{\omega}^{1} u, \quad u=\Psi_{1}(\omega)^{*-1} v
$$

and

$$
K_{\omega}^{2} v=\Psi_{1}^{-1} k_{\omega}^{2} u
$$

are the components of $K_{\omega} v$ with respect to the direct sum decomposition

$$
\mathscr{K}=\mathscr{K}_{1} \dot{+} \mathscr{K}_{2}
$$

Consequently, if $f_{1}$ and $f_{2}$ are the corresponding components of $f \in \mathscr{K}$, then

$$
\begin{aligned}
{\left[f, K_{\omega} v\right]_{\mathscr{K}} } & =-\left\langle f_{1}, K_{\omega}^{1} v\right\rangle+\left\langle f_{2}, K_{\omega}^{2} v\right\rangle \\
& =\left\langle f_{1}, \Psi_{1}^{-1} k_{\omega}^{1} u\right\rangle+\left\langle f_{2}, \Psi_{1}^{-1} k_{\omega}^{2} u\right\rangle \\
& =\left\langle\Psi_{1} f_{1}, k_{\omega}^{1} u\right\rangle+\left\langle\Psi_{1} f_{2}, k_{\omega}^{2} u\right\rangle \\
& =u^{*} \Psi_{1}(\omega) f_{1}(\omega)+u^{*} \Psi_{1}(\omega) f_{2}(\omega) \\
& =v^{*} f(\omega) .
\end{aligned}
$$


This completes the proof of (4).

(5) is established in [1].

We remark that, with the help of Lemma 3.1 , it is readily seen that

$$
\begin{gathered}
\mathscr{K}(\Theta)=\Psi_{1}^{-1}\left\{\mathscr{H}\left(\Psi_{1}\right)+\mathscr{H}\left(\Psi_{2}\right)\right\} \\
\mathscr{K}_{1}(\Theta)=\Psi_{1}^{-1} \mathscr{H}\left(\Psi_{1}\right) \quad \text { and } \quad \mathscr{K}_{2}(\Theta)=\Psi_{1}^{-1} \mathscr{H}\left(\Psi_{2}\right),
\end{gathered}
$$

which is the description which emerges naturally from Theorem 6.6 of [1] when it is specialized to the present setting.

\section{Gram matrices and the distribution of zeros}

In this section we develop another description of the reproducing kernel Krein space $\mathscr{K}(\Theta)$ for $\Theta=N D^{-1}$, when $D$ is a reflection of $N$. We then use two different representations of the reproducing kernel to obtain a formula for the Grammian with respect to an expedient basis for $\mathscr{K}(\Theta)$ in terms of a matrix based on the coefficients of the polynomials $\hat{N}$ and $\hat{D}$.

We begin with two preliminary lemmas.

Lemma 4.1. If $P_{1}$ and $P_{2}$ are left coprime $m \times m$ matrix polynomials, then

$$
H_{m}^{2}+P_{1}^{-1} P_{2} H_{m}^{2}=P_{1}^{-1} H_{m}^{2} .
$$

Proof. For any choice of $f$ and $g$ in $H_{m}^{2}$,

$$
f+P_{1}^{-1} P_{2} g=P_{1}^{-1}\left\{P_{1} f+P_{2} g\right\} \in P_{1}^{-1} H_{m}^{2}
$$

Thus the space on the left hand side of the asserted equality sits inside the space on the right. But, since $P_{1}$ and $P_{2}$ are left coprime there exist a pair of $m \times m$ matrix polynomials $A$ and $B$ such that

$$
P_{1} A+P_{2} B=I_{m}
$$

see e.g. [10]. Therefore, for any $f \in H_{m}^{2}$,

$$
\begin{aligned}
P_{1}^{-1} f & =P_{1}^{-1}\left(P_{1} A+P_{2} B\right) f \\
& =A f+P_{1}^{-1} P_{2} B f \in H_{m}^{2}+P_{1}^{-1} P_{2} H_{m}^{2},
\end{aligned}
$$

which establishes the opposite inclusion.

Lemma 4.2. If $P_{3}$ and $P_{4}$ are right coprime $m \times m$ matrix polynomials, then

$$
H_{m}^{2} \cap\left(P_{3} P_{4}^{-1} H_{m}^{2}\right)=P_{3} H_{m}^{2}
$$


Proof. Suppose first that $f$ belongs to the space on the left hand side of the asserted equality. Then $f \in H_{m}^{2}$ and there exists a $g \in H_{m}^{2}$ such that

$$
f=P_{3} P_{4}^{-1} g \text {. }
$$

But now as $P_{3}$ and $P_{4}$ are right coprime, there exist a pair of $m \times m$ matrix polynomials $F$ and $G$ such that

$$
F P_{3}+G P_{4}=I_{m}
$$

Thus,

$$
\begin{aligned}
f & =P_{3}\left(F P_{3}+G P_{4}\right) P_{4}^{-1} g \\
& =P_{3} F\left(P_{3} P_{4}^{-1}\right) g+P_{3} G g \\
& =P_{3} F f+P_{3} G g \in P_{3} H_{m}^{2} .
\end{aligned}
$$

Now suppose conversely that $f \in P_{3} H_{m}^{2}$. Then $f \in H_{m}^{2}$ and also

$$
f \in P_{3} P_{4}^{-1} P_{4} H_{m}^{2} \subset P_{3} P_{4}^{-1} H_{m}^{2}
$$

Theorem 4.1. Let $N$ be a nonsingular $m \times m$ matrix polynomial with

$$
\operatorname{det} N(\lambda)=a\left(\lambda-\alpha_{1}\right) \ldots\left(\lambda-\alpha_{n}\right)
$$

column degrees $d_{i}, i=1, \ldots, m$, and reflection $D$ and suppose that $\hat{N}$ and $\hat{D}$ are left coprime. Then:

(1) $\hat{N}, N, \hat{D}$ and $D$ are all invertible on $T$.

(2) $\mathscr{K}(\Theta)=\hat{N}^{-1}\left(H_{m}^{2} \ominus \Delta H_{m}^{2}\right)\left(=\left\{f \in L_{m}^{2}: \hat{N} f \in H_{m}^{2} \ominus \Delta H_{m}^{2}\right\}\right)$.

(3) $N$ is a column reduced, i.e., $n=\sum_{j} d_{j}=d$.

Proof. Suppose first (contrary to (1)) that

$$
N(\alpha) \xi=0
$$

for some point $\alpha \in \mathbb{T}$ and some unit vector $\xi \in \mathbb{C}^{m}$. Then, since $N D^{-1}$ is unitary on $\mathbb{T}$,

$$
D(\alpha)^{*} D(\alpha) \xi=N(\alpha)^{*} N(\alpha) \xi=0
$$

But this implies that

$$
N(\alpha) \xi=D(\alpha) \xi=0
$$

which is not consistent with the existence of polynomial matrices $A$ and $B$ such that 


$$
A(\lambda) N(\lambda)+B(\lambda) D(\lambda)=I_{m}
$$

Therefore $N$ is invertible on $\mathbb{T}$, as is $D$ by the very same argument. The rest of (1) is now an easy consequence of the definitions of $\hat{N}$ and $\hat{D}$.

Next, to obtain (2), observe that by assumption

$$
\Theta=\hat{N}^{-1} \hat{D}=N D^{-1},
$$

where the matrix polynomials $N$ and $D$ are right coprime and the matrix polynomials $\hat{N}$ and $\hat{D}$ are left coprime. Thus, by Lemma 4.1 ,

$$
H_{m}^{2}+\Theta H_{m}^{2}=\hat{N}^{-1} H_{m}^{2}
$$

whereas, by Lemma 4.2 ,

$$
H_{m}^{2} \cap \Theta H_{m}^{2}=N H_{m}^{2}
$$

Therefore

$$
\mathscr{K}(\Theta)=\hat{N}^{-1} H_{m}^{2} \ominus N H_{m}^{2}
$$

and hence $f \in \mathscr{K}(\Theta)$ if and only if $\hat{N} f \in H_{m}^{2}$ and $f$ is orthogonal to $N H_{m}^{2}$. But the latter is the same as to say that

$$
\begin{aligned}
0 & =\left\langle f, N_{g}\right\rangle=\left\langle N^{\#} f, g\right\rangle \\
& =\left\langle\Delta N^{\#} f, \Delta g\right\rangle=\langle\hat{N} f, \Delta g\rangle
\end{aligned}
$$

for every $g \in H_{m}^{2}$. Thus $f \in \mathscr{K}(\Theta)$ if and only if

$$
\hat{N} f \in\left(H_{m}^{2} \Theta \Delta H_{m}^{2}\right)
$$

which is equivalent to the asserted description of $\mathscr{K}(\Theta)$ in $(2)$.

Finally, it follows from (2) that $\mathscr{K}(\Theta)$ is a $d$ dimensional space and hence by Theorem 3.1 , that $\Theta$ has MacMillan degree $d=\sum d_{j}$. Thus

$$
\begin{aligned}
& n=\operatorname{deg}\{\operatorname{det} N(\lambda)\} \\
& \geqq \text { the number of elementary factors in } \Theta \\
& \geqq d \geqq n .
\end{aligned}
$$

This proves that equality prevails in the preceding string of inequalities and hence that $N$ is column reduced.

Theorem 4.2. Let $N$ be a nonsingular column reduced $m \times m$ matrix polynomial with 
column degrees $d_{1}, \ldots, d_{m}$ and reflection $D$. Let $\phi_{i}, i=1, \ldots, d$, be a basis for the $d$ dimensional vector space $H_{m}^{2} \ominus \Delta H_{m}^{2}, d=\sum_{i=1}^{m} d_{i}$, let

$$
f_{i}=\hat{N}^{-1} \phi_{i}, \quad i=1, \ldots, d,
$$

and let $G$ denote the Gram matrix of the basis $f_{1}, \ldots, f_{d}$, i.e., the $d \times d$ matrix with $i j$ entry

$$
G_{i j}=\left[f_{j}, f_{i}\right]_{\mathscr{K}}, \quad i, j=1, \ldots, d
$$

Then

$$
\frac{\hat{N}(\lambda) \hat{N}(\omega)^{*}-\hat{D}(\lambda) \hat{D}(\omega)^{*}}{\rho_{\omega}(\lambda)}=\sum_{i, j=1}^{d} \phi_{i}(\lambda)\left(G^{-1}\right)_{i j} \phi_{j}(\omega)^{*}
$$

for every choice of $\lambda$ and $\omega$ in $\mathbb{C}$.

Proof. By Lemma $2.5, \hat{N}$ and $\hat{D}$ are left coprime. Therefore Theorem 4.1 applies and serves to identify the vector functions $f_{1}, \ldots, f_{d}$ as a basis for the reproducing kernel Krein space $\mathscr{K}(\Theta)$. Thus the matrix $G$ is invertible (though not positive definite unless $\Theta$ is analytic and hence contractive in $\mathbb{D}$ ) and it is readily checked by direct calculation that

$$
K_{\omega}(\lambda)=\sum_{i, j=1}^{d} f_{i}(\lambda)\left(G^{-1}\right)_{i j} f_{j}(\omega)^{*}
$$

is a reproducing kernel for $\mathscr{K}(\Theta)$. Therefore, since a reproducing kernel Krein space admits only one reproducing kernel, the right hand sides of the two formulas (3.2) and (4.2) for $K_{\omega}(\lambda)$ must agree:

$$
\frac{I_{m}-\Theta(\lambda) \Theta(\omega)^{*}}{\rho_{\omega}(\lambda)}=\sum_{i, j=1}^{d} f_{i}(\lambda)\left(G^{-1}\right)_{i j} f_{j}(\omega)^{*}
$$

for every choice of $\lambda$ and $\omega$ in $\Omega$, the domain of analyticity of $\Theta$ in $\mathbb{D}$. Formula (4.1) now drops out upon multiplying through by $\hat{N}(\lambda)$ on the left and $\hat{N}(\omega)^{*}$ on the right and invoking (2) of Lemma 2.5 .

To apply Theorem 4.2 we must specify a basis for $H_{m}^{2} \ominus \Delta H_{m}^{2}$. In a self-explanatory notation,

$$
H_{m}^{2} \ominus \Delta H_{m}^{2}=\left[\begin{array}{c}
H^{2} \ominus \lambda^{d_{1}} H^{2} \\
\vdots \\
H^{2} \ominus \lambda^{d_{m}} H^{2}
\end{array}\right]
$$

Thus $H_{m}^{2} \Theta \Delta H_{m}^{2}$ consists of those $m \times 1$ vector polynomials whose $j$ th component is a 
polynomial of degree less than $d_{j}$. There is an obvious basis for this space. Define $e_{c} \otimes \lambda^{p}, 1 \leqq c \leqq m, p=0,1,2, \ldots$, to be the $m \times 1$ vector polynomial with $\lambda^{p}$ in the $c$ th component and zeros in the remaining ones. A basis for $H_{m}^{2} \ominus H_{m}^{2}$ is clearly $\left\{e_{c} \otimes \lambda^{p}\right.$ : $\left.1 \leqq c \leqq m, 0 \leqq p<d_{c}\right\}$. We take this to be the basis $\phi_{1}, \ldots, \phi_{d}$, with the ordering as a subset of

$$
e_{1} \otimes \lambda^{0}, e_{2} \otimes \lambda^{0}, \ldots, e_{m} \otimes \lambda^{0}, e_{1} \otimes \lambda^{1}, e_{2} \otimes \lambda^{1}, \ldots, e_{m} \otimes \lambda^{k-1} .
$$

We shall refer to the corresponding basis of $\mathscr{K}(\Theta)$ :

$$
\hat{N}^{-1} \phi_{1}, \ldots, \hat{N}^{-1} \phi_{d}
$$

as the expedient basis of $\mathscr{K}(\Theta)$.

Theorem 4.3. Let $N$ be a nonsingular column reduced $m \times m$ matrix polynomial with column degrees $d_{1}, \ldots, d_{m}$ and reflection $D$. Then the Gram matrix $G$ of the expedient basis of $\mathscr{K}(\Theta)$ is given by

$$
G=\left(X X^{*}-Y Y^{*}\right)^{-1}
$$

where $X, Y$ are the submatrices of the block Toeplitz matrices described in Theorem 1.1.

Proof. This is essentially a restatement of Theorem 4.2 for the expedient basis: the difficulty of extracting the Gram matrix form is primarily notational. We have to regard $G^{-1}$ as a block matrix. The $\phi_{j}$ 's naturally fall into $k\left(=\max _{j} d_{j}\right)$ blocks, numbered 0 to $k-1$, the elements of block $p$ (say $\phi_{i_{\mathrm{p}}}, \phi_{i_{\mathrm{p}}+1}, \ldots, \phi_{i_{\mathrm{p}+1-1}}$ ) being those of the vector functions $e_{c} \otimes \lambda^{p}, 1 \leqq c \leqq m$, for which $d_{c}>p$. Let us say that an index $c$ is live in the $p$ th block if $d_{c}>p$ and dead otherwise. Let $E_{p}$ be the matrix obtained from the identity matrix $I_{m}$ by deleting all rows whose indices are dead in the $p$ th block. $E_{p}$ is of type $m_{p} \times m$, where $m_{p}$ is the number of live indices in the pth block. $G^{-1}$ can be regarded (in accordance with this division of the basis) as a $k \times k$ block matrix, its $p q$-block ( $g^{p q}$, say) being of type $m_{p} \times m_{q}$. Let us also write $G^{-1}=\left[\gamma_{i j}\right], i, j=1, \ldots, d$.

The right hand side of (4.1) is an $m \times m$ matrix polynomial in $\lambda$ and $\omega^{*}$. Let $A_{p q}$ denote the sum of those terms corresponding to values of $i$ and $j$ such that $\phi_{i}$ and $\phi_{j}$ belong to the $p$ th and $q$ th blocks of the basis respectively:

$$
\begin{aligned}
A_{p q} & =\sum_{s=i_{p}}^{i_{p+1}-1} \sum_{t=i_{q}}^{i_{q}+1-1} \phi_{s}(\lambda) \gamma_{s t} \phi_{t}(\omega)^{*} \\
& =\lambda^{p}\left\{E_{p}^{*} g^{p q} E_{q}\right\} \omega^{* q} .
\end{aligned}
$$

Therefore, since

$$
g^{p q}=E_{p}\left\{E_{p}^{*} g^{p q} E_{q}\right\} E_{q}^{*}
$$


it follows from (4.1) and (4.3) that $g^{p q}$ can be obtained by taking the coefficient of $\lambda^{p} \omega^{* q}$ in the left hand side of (4.1), premultiplying by $E_{p}$ and postmultiplying by $E_{q}^{*}$. Now from (1.1) we have

$$
\begin{aligned}
& \hat{N}(\lambda) \hat{N}(\omega)^{*} / \rho_{\omega}(\lambda) \\
& \quad=\left(N_{0}^{*}+N_{1}^{*} \lambda+\cdots+N_{k}^{*} \lambda^{k}\right)\left(N_{0}+N_{1} \omega^{*}+\cdots+N_{k} \omega^{* k}\right)\left(1+\lambda \omega^{*}+\lambda^{2} \omega^{* 2}+\cdots\right)
\end{aligned}
$$

and so the coefficient of $\lambda^{p} \omega^{* q}(0 \leqq p, q \leqq k-1)$ is

$$
N_{p}^{*} N_{q}+N_{p-1}^{*} N_{q-1}+N_{p-2}^{*} N_{q-2}+\cdots
$$

A similar calculation applies to $\hat{D}(\lambda) \hat{D}(\omega)^{*} / \rho_{\omega}(\lambda)$, and so the coefficient of $\lambda^{p} \omega^{* q}$ in (4.1) is

$$
\sum_{0 \leqq j \leqq \min (p, q)} N_{p-j}^{*} N_{q-j}-D_{p-j}^{*} D_{q-j}
$$

Thus

$$
g^{p q}=E_{p}\left[\begin{array}{lll}
N_{p}^{*} N_{p-1}^{*} \ldots N_{0}^{*} & 0 \ldots 0
\end{array}\right]\left[\begin{array}{c}
N_{q} \\
N_{q-1} \\
\vdots \\
N_{0} \\
0 \\
\vdots \\
0
\end{array}\right] E_{q}^{*}-E_{p}\left[\begin{array}{lllll}
D_{p}^{*} & D_{p-1}^{*} \ldots D_{0}^{*} & 0 \ldots 0
\end{array}\right]\left[\begin{array}{c}
D_{q} \\
D_{q-1} \\
\vdots \\
D_{0} \\
0 \\
\vdots \\
0
\end{array}\right] E_{q}^{*} .
$$

Hence

where

$$
G^{-1}=\left[g^{p q}\right]_{p, q=0}^{k-1}=X X^{*}-Y Y^{*}
$$

$$
X=\left[\begin{array}{cccc}
E_{0} & 0 & \ldots & 0 \\
0 & E_{1} & \ldots & 0 \\
\vdots & \vdots & \vdots & \vdots \\
0 & 0 & \ldots & E_{k-1}
\end{array}\right]\left[\begin{array}{cccc}
N_{0}^{*} & 0 & \ldots & 0 \\
N_{1}^{*} & N_{0}^{*} & \ldots & 0 \\
\vdots & \vdots & \vdots & \vdots \\
N_{k-1}^{*} & N_{k-2}^{*} & \ldots & N_{0}^{*}
\end{array}\right],
$$

with a similar expression for $Y$ in terms of the $D_{j}^{*}$. This agrees with the description of $X$ and $Y$ in Theorem 1.1 and so proves Theorem 4.3.

Proof of Theorem 1.1. Suppose first that the zeros of $N(\lambda)$ are non-conjugate with 
respect to $\pi$ and that $N(\lambda)$ has $p$ zeros inside $\pi$ and $q$ zeros outside, so that $p+q=d=\sum_{j} d_{j}$, and let $D$ be a reflection of $N$. Then, by (4) and (3) of Lemma 2.6, Lemma 2.3 is applicable; it guarantees that $N D^{-1}=\Theta$ is of the form (2.1) with $n=d$. We shall suppose that the zeros of $N$ are ordered so that $\alpha_{1}, \ldots, \alpha_{q}$ are outside $T$ and $\alpha_{q+1}, \ldots, \alpha_{d}$ inside. Let

$$
\Psi_{1}=\left\{V_{0} B_{1} V_{1} \ldots B_{q} V_{q}\right\}^{-1}=V_{q}^{*} B_{q}^{-1} \ldots V_{1}^{*} B_{1}^{-1} V_{0}^{*}
$$

and

$$
\Psi_{2}=B_{q+1} V_{q+1} \ldots B_{d} V_{d}
$$

where $B_{j}$ is short for $B_{\alpha_{j}}$. Then $\Psi_{1}$ and $\Psi_{2}$ are (inner) Blaschke-Potapov products of length $q$ and $p$ respectively which are left inner coprime since the space $\mathscr{K}(\Theta)$ based on $\Theta=\Psi_{1}^{-1} \Psi_{2}$ is $d$ dimensional. We may therefore apply Theorem 3.1 to construct the indefinite inner product space

$$
\mathscr{K}(\Theta)=\mathscr{K}_{1}+\mathscr{K}_{2}
$$

where $\mathscr{K}_{j}$ is linearly isomorphic to $\mathscr{H}\left(\Psi_{j}\right)$. By Lemma $3.1, \mathscr{K}_{1}$ and $\mathscr{K}_{.2}$ have dimensions $q$ and $p$ respectively.

It follows that the Gram matrix of any basis of $\mathscr{K}(\Theta)$ with respect to $[\text {, }]_{\mathscr{X}}$ has $p$ positive and $q$ negative eigenvalues. (For, since the Gram matrices of any pair of bases are congruent, it suffices to prove the assertion for a single basis. Choose orthonormal bases $x_{1}, \ldots, x_{q}$ and $x_{q+1}, \ldots, x_{d}$ in $\mathscr{K}_{1}, \mathscr{K}_{2}$ respectively with respect to $\langle.,$.$\rangle . Then$ $x_{1}, \ldots, x_{d}$ is a basis of $\mathscr{K}(\Theta)$ and its Gram matrix with respect to $[,]_{\mathscr{X}}$ has the form

$$
\left[\begin{array}{cc}
I_{p} & C \\
C^{*} & -I_{q}
\end{array}\right]=\left[\begin{array}{cc}
I_{p} & 0 \\
C^{*} & \left(I_{q}+C^{*} C\right)^{\frac{1}{2}}
\end{array}\right]\left[\begin{array}{cc}
I_{p} & 0 \\
0 & -I_{q}
\end{array}\right]\left[\begin{array}{cc}
I_{p} & C \\
0 & \left(I_{q}+C^{*} C\right)^{\frac{1}{2}}
\end{array}\right]
$$

for some $p \times q$ matrix $C$, and so does have $p$ positive and $q$ negative eigenvalues.)

Finally, we apply the foregoing assertion to the expedient basis of $\mathscr{K}(\Theta)$. By Theorem 4.3 its Gram matrix is $\left(\mathrm{XX}^{*}-\mathrm{Y} \mathrm{Y}^{*}\right)^{-1}$, and so this matrix has $p$ positive and $q$ negative eigenvalues. This completes the proof of Theorem 1.1 when the zeros of $N$ are nonconjugate. To obtain the general case, observe first that (4.1) can be re-expressed in the form (11.1) of [12]:

$$
X(\lambda) J X(\omega)^{*}=\rho_{\omega}(\lambda) Y(\lambda) \Gamma Y(\omega)^{*},
$$

where $Y=\left[\phi_{1} \ldots \phi_{d}\right]$ is the $m \times d$ matrix with columns $\phi_{1}, \ldots, \phi_{d}, \Gamma=G^{-1}$,

$$
J=\left[\begin{array}{cc}
I_{m} & 0 \\
0 & -I_{m}
\end{array}\right] \text { and } X=\left[\begin{array}{ll}
\hat{N} & \hat{D}
\end{array}\right]
$$


Let $\delta_{+}(P)$ [resp. $\left.\delta_{-}(P)\right]$ denote the number of zeros of the matrix polynomial $P$ inside $\mathbb{D}$ [resp. outside $\overline{\mathbb{D}}]$ and let $\mu_{+}(A)$ [resp. $\left.\mu_{-}(A)\right]$ denote the number of correspondingly positioned eigenvalues of the matrix $A$. Then, since $\hat{N}$ and $\hat{D}$ are invertible on $T$, it follows from Lemma 11.1 of [12] that

$$
\delta_{+}(\hat{N})=\left.\operatorname{dim} \operatorname{ker} \mathbf{p} \hat{N}^{*}\right|_{H_{m}^{2}}
$$

where p denotes the orthogonal projection of $L_{m}^{2}$ onto $H_{m}^{2}$. Let $g_{1}, \ldots, g_{v}$ be a basis for the kernel of the indicated operator. Then, just as in the proof of Theorem 11.1 of [12], there is an associated system of vectors $u_{1}, \ldots, u_{v}$ in $\mathbb{C}^{d}$ such that

$$
\left\{\sum_{s=1}^{v} b_{s} u_{s}\right\}^{*} G^{-1}\left\{\sum_{t=1}^{v} b_{t} u_{t}\right\}=-\left\|\mathbf{p} \hat{D}^{*} \sum_{s=1}^{v} b_{s} g_{s}\right\|^{2}
$$

for every choice of the complex constants $b_{1}, \ldots, b_{v}$, other than all zero. But now as $\hat{N}$ and $\hat{D}$ are left coprime, there exist a pair of matrix polynomials $A$ and $B$ such that

$$
\hat{N}(\lambda) A(\lambda)+\hat{D}(\lambda) B(\lambda)=I_{m}
$$

for every $\lambda \in \mathbb{C}$. Therefore

$$
\begin{aligned}
g & =\mathbf{p} A^{*} \hat{N}^{*} g+\mathbf{p} B^{*} \hat{D}^{*} g \\
& =\mathbf{p} A^{*} \mathbf{p} \hat{N}^{*} g+\mathbf{p} B^{*} \mathbf{p} \hat{D}^{*} g,
\end{aligned}
$$

which in turn implies that $\mathbf{p} \hat{N}^{*} g$ and $\mathbf{p} \hat{D}^{*} g$ cannot both vanish for a nonzero $g \in H_{m}^{2}$. Thus the right hand side of (4.4) is strictly positive for every nonzero choice of the constants $b_{1}, \ldots, b_{v}$. This proves that the vectors $u_{1}, \ldots, u_{v}$ span a $v$ dimensional space in $\mathbb{C}^{d}$ which is negative with respect to the inner product induced by $G^{-1}$. Thus

$$
\delta_{+}(\widehat{N}) \leqq \mu_{-}\left(G^{-1}\right)
$$

and similarly

$$
\delta_{+}(\hat{D}) \leqq \mu_{+}(G-1)
$$

The next step is to deduce the formula

$$
\frac{D^{0}(\lambda) D^{0}(\omega)^{*}-N^{0}(\lambda) N^{0}(\omega)^{*}}{\rho_{\omega}(\lambda)}=\frac{1}{\lambda \omega^{*}} \sum_{i, j=1}^{d} \Delta(\lambda) \phi_{i}(1 / \lambda)\left(G^{-1}\right)_{i j} \phi_{j}(1 / \omega)^{*} \Delta(\omega)^{*}
$$

from (4.1) by replacing $\lambda$ by $1 / \lambda, \omega$ by $1 / \omega$, multiplying through by $\Delta(\lambda)$ on the left and $\Delta(\omega)^{*}$ on the right and then setting $N^{0}(\lambda)=N\left(\lambda^{*}\right)^{*}$ and $D^{0}(\lambda)=D\left(\lambda^{*}\right)^{*}$. Much of the same sort of analysis as was used before then leads to the supplementary pair of inequalities 


$$
\begin{gathered}
\delta_{+}\left(D^{0}\right) \leqq \mu_{-}\left(G^{-1}\right) \\
\delta_{-}\left(N^{0}\right) \leqq \mu_{+}\left(G^{-1}\right) .
\end{gathered}
$$

But now as

$$
\delta_{+}\left(N^{0}\right)=\delta_{+}(N) \quad \text { and } \quad \delta_{+}(\hat{N})=\delta_{-}(N)
$$

it follows that

$$
d=\delta_{+}(N)+\delta_{-}(N) \leqq \mu_{+}(G)+\mu_{-}(G)=d .
$$

Therefore equality must prevail in (4.5) and (4.8) as needed.

We remark that since $\hat{D}$ has $d$ zeros and $\delta_{+}\left(D^{0}\right)=\delta_{+}(D)=\delta_{-}(\hat{D})$, equality must prevail in (4.6) and (4.7) also and hence we also have

$$
\delta_{ \pm}(\hat{D})=\mu_{ \pm}\left(G^{-1}\right)
$$

Acknowledgement. We are grateful to the Edinburgh Mathematical Society Centennial Fund for providing financial support for this work. H. Dym also wishes to thank Renee and Jay Weiss for endowing the chair which supports his research.

\section{REFERENCES}

1. D. Alpay and H. Dym, On applications of reproducing kernel spaces to the Schur algorithm and rational $J$ unitary factorization, in: $I$. Schur Methods in Operator Theory and Signal Processing (Operator Theory: Advances and Applications, OT 18, Birkhäuser-Verlag, Basel, 1986), 89-159.

2. B. D. O. Anderson and E. I. Jury, Generalized Bezoutian and Sylvester matrices in multivariable linear control, IEEE Trans. Automat. Control 21 (1976), 551-556.

3. J. A. Ball, I. C. Gohberg and L. Rodman, Realization and interpolation of rational matrix functions, in: Topics in Interpolation Theory of Rational Matrix-valued Functions (Operator Theory: Advances and Applications, OT 33, Birkhäuser-Verlag, Basel, 1988), 1-72.

4. A. ConN, Über die Anzahl der Wurzeln einer algebraischen Gleichung in Einem Kreise, Math. Z. 14 (1922), 110-148.

5. P. Dewilde, Input-output description of roomy systems, SIAM J. Control Optim. 14 (1976), 712-736.

6. P. A. Funrmann, On the corona theorem and its applications to spectral problems in Hilbert space, Trans. Amer. Math. Soc. 132 (1968), 55-66.

7. I. Gohberg, P. Lancaster and L. Rodman, Matrix Polynomials (Academic Press, New York, 1982).

8. T. Kallath, Linear Systems (Prentice Hall, Englewood Cliffs, New Jersey, 1980).

9. L. Lerer and M. Tismenetsky, The Bezoutian and the eigenvalue-separation problem for matrix polynomials, Integral Equations Operator Theory 5 (1982), 386-445. 
10. C. C. MACDuffee, The Theory of Matrices (Springer, Berlin 1933; reprinted by Chelsea Publishing Co., New York, 1950).

11. I. SCHUR, Über potenzreihen, die im Innern des Einheitskreises beschränkt sind, J. für Mathematik 147 (1917), 205-232 and 148 (1918), 122-145; English Transl. in: I. Schur Methods in Operator Theory and Signal Processing (Operator Theory: Advances and Applications, OT 18, Birkhäuser-Verlag, Basel, 1986), 31-88.

12. H. Dym, Hermitian block Toeplitz matrices, orthogonal polynomials, reproducing kernel Pontryagin spaces, interpolation and extension, in: Orthogonal Matrix-valued Polynomials and Applications (Operator Theory: Advances and Applications, OT 34, Birkhäuser-Verlag, Basel, 1988), 79-135.

Department of Theoretical Mathematics

The Weizmann Institute of Science

Rehovot 76100, IsRael

Department of Mathematics

UNIVERSITY OF LANCASTER

LANCASTER LAI 4YF, U.K. 